\title{
Clinical improvement after change of therapy from tafamidis to patisiran in progressive TTR amyloidosis post-liver transplantation
}

\author{
Catherine Bulinski ${ }^{1} \odot \cdot$ Thomas Discher $^{1} \cdot$ Wiebke Rutsatz $^{2} \cdot$ Birgit Assmus $^{2} \cdot$ Heidrun H. Krämer ${ }^{3}$
}

Received: 10 December 2021 / Revised: 16 January 2022 / Accepted: 18 January 2022 / Published online: 6 February 2022

(c) The Author(s) 2022

\section{Dear Sirs,}

Hereditary amyloidosis with autosomal-dominant mutations in the transthyretin-gene (TTR) represents a severe multisystem disorder. Treatment options are now available for TTR familial amyloid polyneuropathy (TTR-FAP): patisiran [1], inotersen [2], tafamidis [3] and orthotopic liver transplantation (OLT). It is known that TTR-cardiomyopathy and neurologic symptoms progress in many transplant recipients. This disease progression after transplantation occurs mainly in patients who are late stage with (p.Val50Met) or have a mutation other than (p.Val50Met) [4]. In these cases, the management strategy is unclear. One small study showed stable neuropathy scores during 12-month treatment with Inotersen after OLT [5]. Tafamidis stabilized TTR-FAP symptoms in one woman for 18 months [6]. Patisiran stabilizes neurological symptoms for 12 months after OLT [7]. No data are available, so far, on how to escalate amyloidosis therapy in case of disease progression after OLT during ongoing therapy with one of the approved agents.

We herewith present a case of a young man who had severe TTR-FAP progression after OLT. Tafamidis therapy did not stop disease progression, but the patient showed clinical improvement of the TTR-FAP after switch to patisiran.

A now 44-year-old man was diagnosed with TTR-FAP in 2012 caused by a rare TTR mutation (p.Glu54Gly) at the age of 36 years. The diagnosis occurred early because of his positive family history. Pre-existing conditions included chronic hepatitis B and D. At diagnosis, he

Catherine Bulinski

catherine.bulinski@innere.med.uni-giessen.de

1 Department of Internal Medicine, Infeciology, Justus Liebig University, Klinikstrasse 33, 35392 Giessen, Germany

2 Department of Cardiology, Justus Liebig University, Giessen, Germany

3 Department of Neurology, Justus Liebig University, Giessen, Germany reported autonomic disturbance causing gastrointestinal symptoms (constipation and diarrhoea) and noticed signs of small-fibre involvement (inability to detect temperatures) for approximately 1 year. Physical examination revealed slightly reduced muscle strength in the extensor muscles of the toes, distal hypesthesia in both feet, pallhypesthesia with $4 / 8$ at the first metatarsophalangeal joints and absent achilles tendon reflexes. Nerve conduction studies (NCS) detected absent sensory responses in the lower limbs and decreased compound muscle action potential (CMAP) of the peroneal nerves. The mNIS + 7score was 31 points [1]. Echocardiography revealed an inhomogeneous echotexture of the myocardium, a slightly reduced systolic function with normal radial and impaired longitudinal function and increased septal thickness $(16 \mathrm{~mm})$. A cardiac MRI performed in 2013 confirmed reduced systolic function (ejection fraction 52\%) and septal hypertrophy. Further, it showed a diffuse late gadolinium enhancement with a non-ischaemic pattern as described in cardiac involvement in amyloidosis. The patient was diagnosed with NYHA stadium I-II and sensorimotor axonal polyneuropathy (Coutinho stage 1). Oral therapy with tafamidis was started. A living split-liver transplantation was performed 2013 without any complications. After OLT, tafamidis medication was discontinued. However, the symptoms of the polyneuropathy slowly increased. In spring 2018, tafamidis medication was re-established. By this time, the patient's complaints comprised of weakness in the legs, sensory loss in the feet, paraesthesia in the hands and postprandial hypotonia indicating autonomic neuropathy. Physical examination revealed paresis of the small hand muscles (MRC 4/5), the extensor muscles of the feet (MRC 2/5), areflexia at the lower extremities and pallanaesthesia at the first metatarsophalangeal joints. NCS detected absent sensory responses in all limbs and absent CMAP of the peroneal and significantly reduced CMAP of the tibial nerves (mNIS-7 score: 149.5). Echocardiography showed rather constant hypertrophy of the 
left ventricular septum with thickness of $17 \mathrm{~mm}$ and stable ejection fraction of 55\%, but no diastolic dysfunction. However, the symptoms further advanced until the patient was unable to walk stairs and required permanent assistance while walking due to increasing gait unsteadiness (Coutinho stage 2). At the beginning of 2019, treatment with patisiran was started. At this time, physical examination revealed additional paresis of all small hand muscles (MRC 3/5), flexor muscles of the feet (MRC 4/5) and toes (MRC 3/5) and of the proximal leg muscles (MRC 4/5), areflexia and hypesthesia on the lower arms and legs. NCS showed now absent CMAP in the lower legs and significantly reduced CMAP in the upper limbs. Nerve sonography detected enlarged cross sectional areas of the median nerves and ulnar nerves at the upper arm (mNIS-7 score: 183.5 ). The patient's TTR-FAP symptoms slowly improved during patisiran therapy. After 18 months of treatment, he could walk independently with two dynamic peroneal splints and climb 7-8 stairs with assistance (mNIS-7 score: 172.5). In addition, echocardiography in 2020 revealed a preserved ejection fraction, and no evidence of diastolic dysfunction (normal E/e', only slightly enlarged left atrium with $30.5 \mathrm{ml} / \mathrm{m}^{2}$ ). By cardiac MRI, a septal hypertrophy of $20 \mathrm{~mm}$ was detected, as well as an increased amount of late gadolinium enhancement. Native $\mathrm{T} 1$ and $\mathrm{T} 2$ relaxation time were elevated. Thus, despite preserved left ventricular function, a progressive cardiac involvement, as suggested by increasing septal thickness, cannot be ruled out. However, as a major limitation, echocardiography and MRI were used across different centres, and detailed off-line core-lab analysis was not available.
NCS remained stable compared to the investigation in 2019 (Table 1).

This case provides evidence that change of treatment regimen in patients with disease progression after OLT is a valuable option. One possible explanation for the different therapeutic effect is the different mode of action of tafamidis and patisiran. Whereas tafamidis [3] acts as a TTRstabilator, patisiran inhibits hepatic synthesis of transthyretin [1]. It is known that amyloidosis associated symptoms after OLT progress due to deposition of healthy amyloid on old TTR-amyloid deposits [4]. Since patisiran leads to significant diminished serum TTR levels even after OLT [7], this mechanism might be reduced.

The cardiac amyloidosis showed no change in our study. However, cardiac examinations were performed at multiple centres with different examination protocols, which limits comparability and interpretability of the results. Most importantly, no MRI-derived quantitative baseline septal thickness (2013) was available. However, a dramatic deterioration of cardiac function can be excluded. Although patisiran is not approved in the cardiac indication, previous studies demonstrated positive effects of patisiran on cardiac parameters, although results were very heterogeneous with respect to reductions in MRI-derived extracellular volumes as an indicator of cardiac TRR-amyloid regression of [8]. However, analysis of cardiac parameters in the APOLLO study showed, among other parameters, a reduction of mean left ventricular wall thickness and improved global longitudinal strain [9].

The number of TTR-amyloidosis patients post-OLT will further decrease over the next years. However, in
Table 1 Depicts clinical data of our patient over the observation period

\begin{tabular}{lllll}
\hline & 2012 & 2018 & 2019 & 2020 \\
\hline $\begin{array}{l}\text { Clinical examination } \\
\text { Coutinho stage }\end{array}$ & 1 & & & \\
mNIS7 score & 31 & 2 & 2 & 2 \\
$\begin{array}{l}\text { Nerve conduction studies } \\
\text { Medial nerve, sensory }\end{array}$ & SNAP: $8 \mu \mathrm{V}$ & Absent & Absent & Absent \\
& NCV: $52 \mathrm{~m} / \mathrm{s}$ & & & \\
Medial nerve, motor & CMAP: $11 \mathrm{mV}$ & CMAP: $4.8 \mathrm{mV}$ & CMAP: $0.9 \mathrm{mV}$ & CMAP: $0.6 \mathrm{mV}$ \\
& NCV: $54 \mathrm{~m} / \mathrm{s}$ & NCV: $48 \mathrm{~m} / \mathrm{s}$ & NCV: $44 \mathrm{~m} / \mathrm{s}$ & NCV: $46 \mathrm{~m} / \mathrm{s}$ \\
Sural nerve & Absent & Absent & Absent & Absent \\
Peroneal nerve & CMAP: $2.5 \mathrm{mV}$ & Absent & Absent & Absent \\
Tibial nerve & NCV: $41 \mathrm{~m} / \mathrm{s}$ & & & Absent \\
Cardiological examinations & CMAP: $8.0 \mathrm{mV}$ & CMAP: $0.9 \mathrm{mV}$ & Absent & \\
NYHA stadium & I-II & NCV: $38 \mathrm{~m} / \mathrm{s}$ & & I-II \\
Septal thickness & $16 \mathrm{~mm}$ & & & $20 \mathrm{~mm}$ \\
Ejection fraction & $52 \%$ & $17 \mathrm{~mm}$ & $18 \mathrm{~mm}$ & $71 \%$ \\
\hline
\end{tabular}

SNAP sensory nerve action potential, $N C V$ nerve conduction velocity, CMAP compound muscle action potential 
these patients, gene-silencing therapy might be an option. Although the use of patisiran improved the clinical status of the patient with the rare mutation (p.Glu54Gly), it cannot be concluded that this positive effect will also occur in patients with other TTR mutations. In summary, larger studies are needed to establish evidence-based treatment recommendations for medical therapy of TTR-amyloidosis patients after OLT.

Acknowledgements This research did not receive any specific grant from funding agencies in the public, commercial, or not-for-profit sectors.

Funding Open Access funding enabled and organized by Projekt DEAL.

\section{Declarations}

Conflicts of interest The authors declare that they have no conflict of interest.

Ethics approval This report was written in conformity with the guidelines of the local ethics committee of the Justus-Liebig-University Giessen and is therefore in accordance with the declaration of Helsinki.

Open Access This article is licensed under a Creative Commons Attribution 4.0 International License, which permits use, sharing, adaptation, distribution and reproduction in any medium or format, as long as you give appropriate credit to the original author(s) and the source, provide a link to the Creative Commons licence, and indicate if changes were made. The images or other third party material in this article are included in the article's Creative Commons licence, unless indicated otherwise in a credit line to the material. If material is not included in the article's Creative Commons licence and your intended use is not permitted by statutory regulation or exceeds the permitted use, you will need to obtain permission directly from the copyright holder. To view a copy of this licence, visit http://creativecommons.org/licenses/by/4.0/.

\section{References}

1. Adams D, Gonzalez-Duarte A, O'Riordan WD et al (2018) Patisiran, an RNAi therapeutic, for hereditary transthyretin amyloidosis. N Engl J Med 379:11-21. https://doi.org/10.1056/NEJMo a1716153

2. Benson MD, Waddington-Cruz M, Berk JL et al (2018) Inotersen treatment for patients with hereditary transthyretin amyloidosis. N Engl J Med 379:22-31. https://doi.org/10.1056/NEJMoa1716 793

3. Coelho T, Maia LF, Martins da Silva A et al (2012) Tafamidis for transthyretin familial amyloid polyneuropathy: a randomized, controlled trial. Neurology 79:785-792. https://doi.org/10.1212/ WNL.0b013e3182661eb1

4. Ericzon B-G, Wilczek HE, Larsson M et al (2015) Liver transplantation for hereditary transthyretin amyloidosis: after 20 years still the best therapeutic alternative? Transplantation 99:1847-1854. https://doi.org/10.1097/TP.0000000000000574

5. Moshe-Lilie O, Dimitrova D, Heitner SB et al (2020) TTR gene silencing therapy in post liver transplant hereditary ATTR amyloidosis patients. Amyloid 27:250-253. https://doi.org/10.1080/ 13506129.2020.1784134

6. Romero-Imbroda J, Sagrario-Fustero T, Del Canto-Pérez C (2017) Tafamidis for a transplant patient with transthyretin amyloid polyneuropathy. J Clin Neurol 13:444-446. https://doi.org/10.3988/ jen.2017.13.4.444

7. Muñoz-Beamud F, Coelho T, Gillmore JD et al (2021) 2021 peripheral nerve society virtual event. J Peripher Nerv Syst 26:307-440. https://doi.org/10.1111/jns. 12460

8. Fontana M, Martinez-Naharro A, Chacko L et al (2021) Reduction in CMR derived extracellular volume with patisiran indicates cardiac amyloid regression. JACC Cardiovasc Imaging 14:189-199. https://doi.org/10.1016/j.jcmg.2020.07.043

9. Solomon SD, Adams D, Kristen A et al (2019) Effects of patisiran, an RNA interference therapeutic, on cardiac parameters in patients with hereditary transthyretin-mediated amyloidosis. Circulation 139:431-443. https://doi.org/10.1161/CIRCULATIONAHA.118. 035831 